\title{
PRODUCTION PLANNING AND CONTROL INFORMATION SYSTEM FOR THE ENGINEERING AND MAKE TO ORDER ENVIRONMENT. A VIRTUAL ENTERPRISE APPROACH
}

\author{
Delva Batista de Chambers ${ }^{1}$ \\ Francisco Sastrón Báguena ${ }^{2}$ \\ Miguel Gutiérrez Fernández ${ }^{3}$ \\ ${ }^{I}$ Universidad Tecnológica de Panamá, PANAMA - dbatista@disam.upm.es \\ ${ }^{2}$ DISAM, Universidad Politécnica de Madrid, SPAIN - sastron@etsii.upm.es \\ ${ }^{3}$ Universidad Carlos III de Madrid, SPAIN - mgfernan@ing.uc3m.es
}

\begin{abstract}
The engineering and make-to-order industry (E\&M-T-O) shows some operating features, which shape an extremely complex project-based production planning and control system (PPC). That complexity has as a result a very low level of computerisation and a lack of an adequate explicit information system. Our research outlines the future scenario in which all the companies taking part in large engineering projects may be involved in the short term. The scenario is built up considering the parallelism between the inter-enterprise consortium formed for an engineering project and the current paradigm of virtual enterprise. Then it is proposed a distributed and open PPC information system for the E\&M-T-O environment, mainly characterised by the capability of the integration of all the companies involved in the supply chain.
\end{abstract}

\section{INTRODUCTION}

Nowadays, both the industry and the society are experiencing fast and continuous changes. These changes, as long with the evolution of information technologies (IT), have imposed a transformation process on the organisations, if they want to maintain their competitive edge [2].

The transformation process has not affected every industry sector with the same urgency. While some have experienced drastic changes, others have merely modified their modus operandi. The latter must adjust to the new scenario in the short term. This is the case of the companies involved in major engineering projects. The E\&M-T-O companies belong to this group. These companies are characterised by manufacturing product with a high technological level along with low if not unique production unitary lots. Such features makes too complex the procedures, 
which leads to low level of automation regarding the PPC area. Such a company must address this problem in the short term with a system-oriented approach.

The challenge for such a company is to design its systems as real information systems, not as mere supporting software systems for operative and managerial activities to solve daily problems and routine decision-making. Such systems do not usually provide data enough for decision-making within complex and highly uncertain levels.

We must bear in mind that computer-based automation and information systems are not synonymous, although they are highly related. Computer-based automation refers to implement tools for the different departments (finance, production, etc) in an independent way. If each of these tools has been properly developed, the specific goal of each department will be satisfied, but there will be a lack of integration and co-ordination.

We propose an information system to support the PPC within the E\&M-T-O environment. Such a system must not only satisfy the particular requirements of this environment (information flows, systems integration and actors involvement) but also allow the company to cope with the forthcoming scenario based on the virtual enterprise (VE) paradigm.

We describe in first instance the particular and specific features of the E\&M-T-O environment. Next, we present the VE, which may constitute the new operating scenario for major engineering projects. Once this new scenario has been explained, we describe our proposed information system. As its main feature, it pays special attention to the relationships and interactions among the several actors involved in the PPC system, including those that cross the company border.

\section{PRODUCTION PLANNING AND CONTROL IN THE E\&M-T-O MANUFACTURING ENVIROMENT}

The PPC system in the E\&M-T-O environment is constrained by four operating features. A thorough analysis of these peculiarities allows to identify the precise aspects that an information system within this manufacturing environment must support:

a) Make to order. Customers, frequently engineering companies, order products for which there are no stock and no production planning.

- There is an engineering stage, prior to manufacturing, where drawings, bill of materials, manufacturing process definition, material requirement and buy orders are issued.

- Raw materials are specific for each order, which prevents for setting a stable supply policy.

- As projects differ from one another, there is not enough expertise and data to estimate activities lead-time added to the uncertainty regarding raw materials lead-times.

- The particularities of each product makes it difficult a bill of material based production management, and hence the use of MRP systems.

b) Demand uncertainty. There is a high uncertainty regarding new orders, added to the high variability in the complexity and total lead-time of the projects. 
- The traditional demand forecast techniques cannot be used, making even more difficult the medium/long term planning.

- "The sooner the better" manufacturing policy appears as a result of not being able to foresee what should be manufactured in the short/medium term.

c) Project structure. Orders are approached following the traditional project structure as precedence net.

- The project structure might be advisable to use the traditional project management techniques. However, as manufacturing takes place in a single shop-floor designed as a job-shop, competence for the manufacturing resources become a major aspect.

- There is a matrix organisational structure based in the assignment of Project Managers.

d) Part of a bigger project. Orders are usually, but not necessarily, part of a bigger engineering project.

- Being an activity to be planned within the bigger project (the customer), there is a demand when it comes to achieving the deadlines and quality. Besides, changes in due dates or technical requirements are quite common.

- Regarding the information system, being part of a bigger engineering project implies some particular requirements. Such a system must provide proper interfaces with close elements of the global supply chain, that is, the customer and the suppliers.

\section{THE VIRTUAL ENTERPRISE}

The information flow in manufacturing was traditionally considered only at a local level. Nowadays, due to the use of IT, the information flow has crossed country and continent borders. Data exchange to co-ordinate the interaction between each distant company becomes a vital aspect if manufacturing companies want to be competitive [7]. This challenge demands a new approach to the enterprise information structure. "Local information systems" will be substituted by "global information systems" as global markets replaced local markets. As a result, concepts such as Extended Enterprise (EE) and VE have been coined.

A VE is a multidisciplinary area that demand well-defined concepts and a common terminology [3]. There is not an unique definition of VE. There are several expressions referring to the same concept or addressing different approaches: EE, Supply Chain Management, Electronic Commerce, Cross Border Enterprise, Network of Enterprises, Virtual Co-operation, Agile Manufacturing, etc. All of them may not be synonymous, but somehow address similar concepts.

Among the several definitions of VE in the literature, the three following ones show a major conceptual difference when it comes to the use of IT:

- The National Industrial Information Infrastructure Protocols (NIIIP) defines the $\mathrm{VE}$ as "a temporary consortium of independent member companies which come together to quickly exploit fast-changing world-wide product manufacturing opportunities. VE assemble themselves based on cost effectiveness and product uniqueness without regard for organisation size, geographic location, computing 
environments, technologies deployed, or processes implemented. VE companies share costs, skills, and core competencies which collectively enable them to access global markets with world-class solutions that could not be provided individually".

- [1] defines it as "a temporary network of supply companies, customers and even competitors joined by IT to share skills, costs and access to new markets".

- [5] regards the VE as "a rapidly configured multi-disciplinary network of small, process-specific firms configured to meet a window of opportunity to design \& produce a specific product".

Currently some international projects use the VE (distributed and open information systems) approach trying to link a certain number of companies within the supply chain. Among them we can point out NIIIP, PRODNET II, INCO-SCM+, Sajonia, VEGA and LOGSME.

\section{THE NEW LARGE ENGINEERING PROJECT SCENARIO}

Usually, several and different companies are involved in a large engineering project. In our research we have distinguished three main groups:

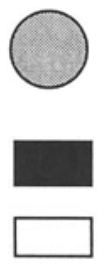

Engineering companies responsible for the whole project. They are usually in charge of subcontracting the activities as well as the co-ordination tasks within the consortium.

E\&M-T-O companies. They make to order heavy equipment as a part of the global project.

Other companies taking part in the consortium as constructors or infrastructure providers.

The underlying concept of the new scenario starts from the existing parallelism between the inter-enterprise consortium formed for a engineering project and that formed as a VE. In both cases, a group of companies establish a temporary bond to achieve a goal.

There are differences between both kinds of consortium depending on the definition of the VE chosen. As aforementioned, there are several definitions that imply fundamental differences such as the role of IT within the VE. However, the discrepancies between the consortia are related mainly to the modus operandi. The traditional consortium shows a strong hierarchy inherited from historical established practices, with a company acting as a leader at the highest level of the hierarchy. The leading company also act as the customer of the subcontracting companies. As opposed, the VE shows a more co-operative operating philosophy, where a company may take the co-ordinating role, although costs, potentials and achievements are still shared. The aim is that the companies complement each other while keeping their own core competencies to achieve a better efficiency as a global result.

The last years trend points out on the one hand that the co-operative aspects are going to be strengthened and on the other hand the increasing important role of the information systems. Therefore, the medium term scenario is shown in Figure.1. Solid lines gather companies belonging to VE consortia while dotted lines represent traditional style consortia. From the figure we can see that the engineering companies, the E\&M-T-O companies and the rest of companies can take part 
simultaneously in different VE. In fact they will in a real scenario. Even, all the companies can belong to traditional style and VE consortia at the same time.

Within this scenario, the information systems play an outstanding role for an adjusted consortium performance. The E\&M-T-O industry would gain better results if the necessary computerisation process previously described would follow the open and distributed systems approach. The design should also consider the possibility of linking to the several temporary information systems constituted for each consortium. In addition, the information system should be flexible enough to operate in traditional style consortia. Those companies approaching the future in this way would gain a competitive advantage, which according to Porter proposal [6], will become a requirement to survive.

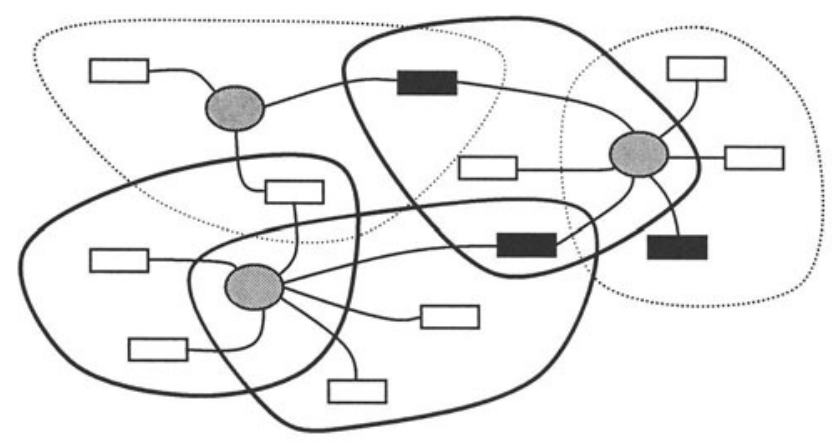

Figure 1 -The new scenario of large engineering projects

\section{INFORMATION SYSTEM FOR PPC IN E\&M-T-O INDUSTRY WITHIN VE FRAMEWORK.}

Features such as information flows related to engineering projects or several enterprises at disperse geographical location interacting with each other, make the E\&M-T-O companies a suitable place to use computer support means to co-ordinate data exchange. To achieve such a co-ordination, any information system supporting PPC must provide the following capabilities:

a) To support any PPC process, which can be grouped according to its nature in three main types:

- Regular processes that occur periodically (e.g. planning and scheduling.)

- Reactive processes such as arrival of urgent projects, machine breakdowns, absenteeism, delays with raw material supplies or longer activity run time.

- Input and output data processes such as machine assignment, resource planning, project data, project status reports.

b) To be able to integrate with existing computer-based systems in the company. On the one hand, this is a main feature required to avoid "information isles" within the company. On the other hand, market available ERP systems follow this trend. 
c) Integration both of the internal (engineering, planning and production departments and project managers) and the external actors (customers, suppliers and outsourcers) involved in the supply chain.

d) To be able to link, in an easy way, to the several temporary information systems constituted for the consortia that decide to work in the VE approach.

Considering the aforementioned features, we propose the information system design shown in Figure 2. Its architecture is currently based on Internet. Nevertheless, a neutral technology could be used to suit other requirements, such as LANs. The following elements can be distinguished:

a) Database server. It is the heart of the information system. A modular approach has been followed to provide for flexibility and integration capabilities. The computer-based tools obtain the specific data from the database, use them in the algorithms and store the updated data in the database (especially, start and finish dates for all activities). Moreover, the database acts as a link/bond with the rest of computer systems in the company. In fact, the database itself could be considered as an ERP system.

b) Web server. It is connected to database server by means of SQL queries (drawn as a solid line). The web server also provides Internet access.

c) Shop-floor computers belonging to the Production Department (drawn as a computer near to a factory symbol). They provide support to usual, reactive and input data processes. User-friendly interfaces allow the users to access the data using SQL by means of a direct link with the web server (drawn as a solid line).

d) Office computers related to Project Managers (if required, part of the Production Department, e.g. Planning, might be included). These computers are directly connected to the database (internal access) or by Internet through the web server (shown as a dashed line).

e) Customers computers (shown as a computer near to a plant symbol). Customers are connected through the web server, which allows them to gather technical data, drawings and to obtain progress reports.

f) Suppliers computers (shown as a computer near to a screw symbol). Suppliers are also connected through the web server, to update their data on raw materials deliveries.

The proposed architecture intends to bring together customers, suppliers, subcontractors (considered as intermediate suppliers) involved in a specific project. All the actors can obtain and notify information on any event that may influence both their own value chain and the E\&M-T-O environment: delays regarding raw material supply, production delays or progress status reports, avoiding in this way the information distortion in the supply chain described in [4]. As an example, a customer (engineering company) could request information on the current status of its order by consulting a web page. After log-in, the customer would obtain a detailed report by queries to the database of the E\&M-T-O company.

Regarding the implementation, some initial try-outs have been carried out to verify the technical viability of the proposed information system. The experiments try to test both the functional and the security aspects related to the system. The latter is a relevant point to consider, taking into account the industry susceptibility when it comes to information confidentiality. 


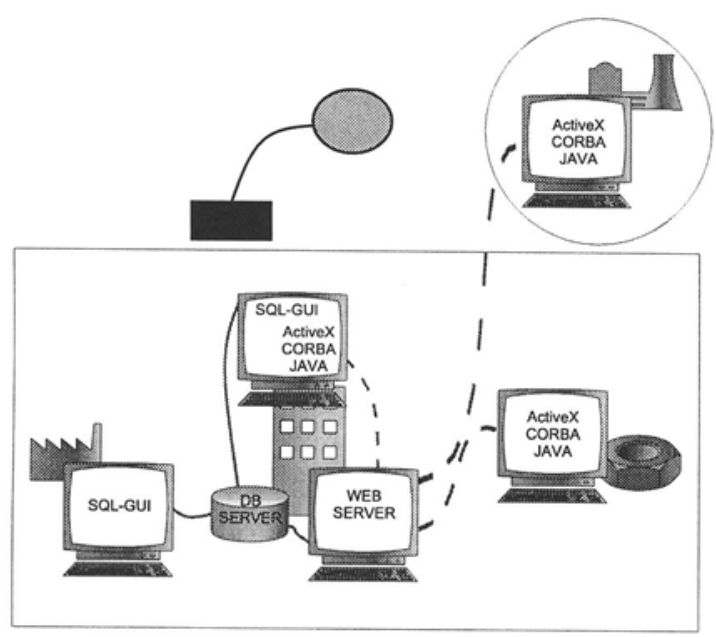

Figure 2 Proposed Information System for PPC in E\&M-T-O Industry

These early prototypes are based on a model where transactions and operations take place in a database using HTML pages by gateways (CGI). Nevertheless, combining HTML-CGI diminishes the graphical capability of the system, reducing its use to well-structured queries. A pre-processing capability at the client side, and additional graphical features are missing in that approach. The researches on Information Technologies suggest distributed objects as the base of future developments.

Following the aforementioned trend, models based on component technology (JavaBeans, ActiveX) are being developed using distributed web sites. Access to objects in a different network node can be made using the paradigm of distributed compound documents based on Microsoft ActiveX or CORBA-OpenDoc [8]. This paradigm allows embedding objects obtained in the network without using browsers. Moreover, the compound document can include methods to edit its content or to run those methods in object server.

In early experiments we have used HTML pages, and transactions performed on a database by means of the Common Gateway Interface (CGI). With regard to security, which is a major requirement of industry, authentication mechanisms have been used to protect the Web server (Apache) and the Database Management System (Minerva).

\section{CONCLUSIONS}

The IT evolution during the last decade has lead to an industrial and economical revolution. New markets have appeared. Market conditions have changed. Companies have to adjust to the changing environment, modifying their operating procedures. As a result of this revolution, new paradigms such as the VE and EE have appeared. In the short term, we consider that companies belonging to the engineering projects sector will evolve to a new scenario based on the VE paradigm in which information systems will play a major role. 
The starting point considers that an engineering project can be approached considering the VE viewpoint, as long as it involves several companies in a temporary way to achieve a goal. The differences between the concept of VE and the underlying idea of an engineering project may differ depending on the definition of VE considered among the existing ones. Regardless the chosen definition, the main contrast is due to the idiosyncrasy of the engineering companies whose modus operandi is based on inherited and expertise-based procedures. As opposed to the co-operative feature of a VE, which implies sharing costs, skills and achievements, companies of current inter-enterprise networks show a rigid hierarchy based on subcontracting. Nevertheless, these differences will fade considering the market trend shown nowadays.

Among the companies affected by this new scenario, we can consider the E\&M-T-O sector, currently characterised by a low automation level in production control, planning and scheduling. Our proposal regarding this essential automation is twofold:

- Firstly, the automation should consider all the information flows regarding both the PPC processes and the internal/external actors. As a requirement, this automation must take place within a distributed and open information system framework.

- Secondly, the information system should be flexible enough to allow its integration with several external information systems to cope with the VE scenario. To be prepared for the new operating way would not only allow to provide for the medium term but also to create an initial competitive advantage to achieve an outstanding position.

\section{ACKNOWLEDGEMENTS}

The authors would like to thank Julia Bermejo, assistant lecturer at Alfonso X University of Madrid, for her valuable suggestions and review work.

\section{REFERENCES}

1. Bienert, A. Computer Aided Enterprise Modelling. A global approach. Advanced in Production. Management Systems, Elsevier Science, 1993.

2. Browne, J., Sackett, J.C., Wortmann, J.C. Future Manufacturing Systems - Towards the Extended Enterprise. Computers in industry, 25: 235 - 254, 1995.

3. Camarinha-Matos, Luis M., Afsarmanesh. H. The virtual enterprise concept. Proceedings of PROVE'99 - IFIP International Conference on Infrastructures for Virtual Enterprises, Kluwer Academic Publishers, 1999.

4. Lee, H.L. Information Distortion in a Supply Chain: The Bullwhip Effect, et. al. Management Science, 43(4): 546 - 558, 1997.

5. Parunak, Van. Technologies for Virtual Enterpirses: A Proposal for a NIST-ATP. NTMS National Center for Manufacturing Sciences, 1994.

6. Porter, M.E. Competitive Advantage. The Free Press, 1985.

7. Richards, H.D., Dudenhausen, H.M., Makatsoris, C., De Ridder, L. Flow of orders through a virtual enterprise: their proactive planning and scheduling and reactive control.. Computing \& Control Engineering Journal, 1997.

8. Van Soest and Verkuil. The Best of Three Worlds. Building a Distributed Application as a Step Toward Business Objects. Bell Labs Technical Journal, 200 - 219, Winter 1997 